\title{
Drug Susceptibility Pattern of Mycobacterium tuberculosis isolates from Extrapulmonary Tuberculosis Cases in Puducherry and Surrounding Tamil Nadu, South India
}

\author{
Gandhi Kandhakumari and Selvaraj Stephen*" \\ Department of Microbiology, Mahatma Gandhi Medical College and Research Institute, \\ SBV University, Pillaiyarkuppam, Pondicherry - 607403, India \\ *Corresponding author
}

\begin{tabular}{|c|c|}
\hline & A B S T R A C T \\
\hline & $\begin{array}{l}\text { Diagnosis of Extrapulmonary tuberculosis (EPTB) remains cumbersome due to non- } \\
\text { specific clinical presentation and paucibacillary nature of samples. Irreversible } \\
\text { complications are common if not diagnosed and treated earlier. Successful treatment }\end{array}$ \\
\hline Keywords & $\begin{array}{l}\text { outcome of EPTB and control of emerging drug resistance depends on adherence to } \\
\text { treatment based on drug susceptibility result. Objective of this research work was to study }\end{array}$ \\
\hline ATT drugs, Drug & drug susceptibility pattern of M. tuberculosis isolates from EPTB specimen. Among 649 \\
\hline $\begin{array}{l}\text { susceptibility } \\
\text { testing, EPTB, } \\
\text { M.tuberculosis. }\end{array}$ & $\begin{array}{l}\text { EPTB patients screened in a tertiary care teaching hospital for a period of over three years, } \\
71 \text { strains }(10.9 \%) \text { of } M \text {. tuberculosis complex (MTBC) were isolated and identified using }\end{array}$ \\
\hline Article Info & n MGIT960 system to first line ATT drugs. Sensitivity to all drugs \\
\hline $\begin{array}{l}\text { Accepted: } \\
\text { 28 September } 2017 \\
\text { Available Online: } \\
10 \text { October } 2017\end{array}$ & $\begin{array}{l}\text { and Isoniazid in } 2.81 \% \text {. Monoresistance to PZA, Isoniazid, Streptomycin, Rifampicin and } \\
\text { Ethambutol was observed in } 9.9 \%, 5.6 \%, 4.2 \%, 2.8 \% \text {, and } 2.8 \% \text { respectively. } \\
\text { Monoresistance of, } 18.3 \% \text { from pus, } 4.2 \% \text { from pleural fluid and } 1.4 \% \text { each from ascitic } \\
\text { fluid and urine respectively was encountered. None of the isolates belonged to MDR-TB }\end{array}$ \\
\hline & $\begin{array}{l}\text { Our study reveals the absence of MDR from new extrapulmonary tuberculosis cases in this } \\
\text { part of India. }\end{array}$ \\
\hline
\end{tabular}

\section{Introduction}

India is one among the countries with high tuberculosis (TB) and multidrug resistant tuberculosis (MDR-TB) burden. It accounts for $23 \%$ of the total 9.6 million incident cases of tuberculosis and 2.2\% of MDR-TB reported globally (WHO, 2015). Tuberculosis is a major health issue in developed and developing countries. It infects both pulmonary and extra pulmonary sites (TB lymph node, pleura, abdomen, skin, etc) of the body. In India, EPTB accounts for 15-
$20 \%$ and 50\% among immune-competent and HIV individuals respectively (Sharma and Mohan, 2004). Due to nonspecific clinical presentation, paucibacillary clinical specimens and lack of appropriate diagnostic tool, most of the patients are treated empirically with first line anti-tuberculous drugs and suspected for drug resistance only when patients fail to recover. This leads to the emergence of drug resistance and progression of the disease condition with treatment delay. 
Today primary drug resistance has been increasingly reported from extrapulmonary tuberculosis cases. (Dusthackeer et al., 2005; Maurya et al., 2012; Yadav et al., 2016; Mittal et al., 2014). This primary drug resistance in EPTB cases may be due to infection with primary drug resistant strains spread out by pulmonary tuberculosis patients. Though EPTB do not spread from one patient to the other posing a threat of spread with primary drug resistant strains, it causes serious complication of the ill patient, unless diagnosed and treated earlier. Nowadays most of the extrapulmonary tuberculosis cases are treated without drug susceptibility testing following the regimen used for treating pulmonary tuberculosis patients except tuberculous meningitis and bone and joint tuberculosis. For effective treatment of the drug resistant tuberculosis cases, modification of the treatment regimen according to the drug susceptibility pattern of the particular strain is needed. Hence this study was undertaken to understand the drug susceptibility pattern of Mycobacterium tuberculosis isolated from EPTB specimens in this region of South India.

\section{Materials and Methods}

Our Institutional Human Ethical Committee (IHEC) has approved this work and informed consent was obtained from patients before collecting the specimen. Between June, 2011 and October, 2014, 649 single EPTB samples collected from patients with clinical/radiological suspicion were processed. Patients who were treated with ATT drugs were excluded from our study. Specimens collected from sterile sites were centrifuged and the deposit inoculated onto Lowenstein Jensen (LJ) slants and automated mycobacterial growth indicator test system (MGIT960). Specimens from unsterile areas were first decontaminated using $\mathrm{NaOH}-$ NALC (Sodium hydroxide -N-acetyl-L- cysteine) procedure. These specimens were inoculated and monitored for eight and six weeks on LJ and MGIT960 respectively. Identification and confirmation of $M$. tuberculosis complex (MTBC) was by standard conventional and MPT64 antigen detection tests as described earlier (Kandhakumari and Stephen, 2015). Drug susceptibility testing was carried out for five first line drugs in MGIT following the recommended procedure of the manufacturer at a final concentration of Streptomycin $1.0 \mu \mathrm{g} / \mathrm{ml}$, Isoniazid $0.1 \mu \mathrm{g} / \mathrm{ml}$, Rifampin $1.0 \mu \mathrm{g} / \mathrm{ml}$, Ethambutol $5 \mu \mathrm{g} / \mathrm{ml}$ and Pyrazinamide $100 \mu \mathrm{g} / \mathrm{ml}$. Briefly $0.8 \mathrm{ml}$ of the SIRE supplement was added to the labeled BACTEC MGIT tubes and $0.1 \mathrm{~mL}$ of the appropriate drug solution was added, which resulted in the desired concentration of a drug in the medium. This was followed by inoculation of $0.5 \mathrm{~mL}$ bacterial suspension. For growth control, the inoculum was diluted to $1: 100$. The inoculated tubes were incubated and monitored by the MGIT system every one hour for increase in fluorescence. For pyrazinamide sensitivity MGIT tubes were incubated for a maximum of three weeks, whereas for the rest of the drugs it was only up to two weeks. Reference strain of $M$. tuberculosis $\mathrm{H} 37 \mathrm{Rv}$ was used as control.

\section{Results and Discussion}

Out of 649 EPTB samples processed, 71 MTBC (70 M. tuberculosis and one isolate of $M$. bovis) isolates grew in LJ and/or MGIT culture. Antimycobacterial susceptibility testing results of 71 MTBC isolates is documented in Table-1. Fifty one isolates $(71.83 \%)$ were sensitive to all the five ATT drugs. Eighteen isolates $(25.35 \%)$ showed mono resistance and two isolates $(2.81 \%)$ showed dual resistance to INH + STR. PZA resistance was seen in maximum number of isolates (7) (9.9\%), followed by $\mathrm{INH}$ resistance in four, STR resistance in 3, RIF 
and EMB resistance in two isolates each. Two strains were resistant to rifampicin but sensitive to INH. None of our isolates showed multi drug resistance (MDR-TB). We have observed monoresistance of, $18.3 \%$ from pus, $4.2 \%$ from pleural fluid and $1.4 \%$ each from ascitic fluid and urine respectively.

Table.1 Drug resistance pattern of MTBC isolates $(n=71)$

\begin{tabular}{|l|l|l|l|}
\hline Sl.No & Patterns of Drug resistance & Total numbers & \% \\
\hline 1 & Pan-sensitive & 51 & 71.83 \\
\hline 2 & Resistance & 20 & 28.17 \\
\hline \multirow{8}{*}{} & a. PZA resistance & 7 & 9.9 \\
\cline { 2 - 4 } & b. Isoniazid resistance & 4 & 5.6 \\
\cline { 2 - 4 } & c. Streptomycin resistance & 3 & 4.2 \\
\cline { 2 - 4 } & d. Rifampicin resistance & 2 & 2.8 \\
\cline { 2 - 4 } & e. Ethambutol resistance & 2 & 2.8 \\
\cline { 2 - 4 } & f. Streptomycin +Isoniazid resistance & 2 & 2.8 \\
\cline { 2 - 4 } & Total & 71 & 100 \\
\hline
\end{tabular}

In the present study, overall resistance of $28.17 \%$ to one or more drugs was observed in EPTB isolates. This is in contrast to report from the same geographical region by NIRT (National Institute of Research in Tuberculosis), Chennai, Tamil Nadu, where $59 \%$ resistance was observed (Dusthackeer et al., 2005). However, the authors themselves have clarified that the sample selection could have been biased, since the physicians referred only those chronically ill patients and non-responders to this central facility under Indian Council of Medical Research (ICMR). From Lucknow, Uttar Pradesh (Maurya et al., 2012) reported a resistance of around $38 \%$. In 2002, Sachdeva et al., reported $50 \%$ monoresistance. Though various authors from India have reported the increasing drug resistance among EPTB cases, (Lee et al., 2015) from Korea reported no significant difference in resistance among the pulmonary and extrapulmonary tuberculosis cases.

According to (Dusthackeer et al., 2005) monoresistance to first line ATT drugs was $27.5 \%$, whereas in our study it is $25.4 \%$ and a lower rate of $17.8 \%$ in Lucknow (Maurya et al., 2012). Gupta et al., 2016 reported increasing streptomycin resistance from $5.5 \%$ in the year 2002-05 to $33 \%$ during the year 2009-2012. Sachdeva et al., in 2002 from Delhi reported the highest prevalence of streptomycin resistance followed by resistance to Isoniazid, Rifampicin and Ethambutol. This highlights the increasing streptomycin resistance during the recent years. Isoniazid resistance with or without additional resistance to other drugs was $30 \%$ in Chennai and $27.6 \%$ at Lucknow (Dusthackeer et al., 2005; Maurya et al., 2012). But we have observed only $8.4 \% \mathrm{INH}$ resistance. Gupta et al., 2016 from Delhi reported isoniazid resistance of $33.3 \%$ during the year 2002-05, which jumped to $60 \%$ during 2009-2012 (Gupta et al., 2016). Regarding rifampicin resistance, it was $24.6 \%$ from NIRT, Chennai and 14\% from New Delhi, (Dusthackeer et al., 2005; Gupta et al., 2016) while we had only a low $2.8 \%$. Sachdeva et al., 2002 from Delhi did not observe any pyrazinamide resistance.

About $2.8 \%$ of our isolates were susceptible to rifampicin but with INH resistance and 
from Lucknow $3.25 \%$ of such cases were reported. These isolates could have been under- reported for INH resistance, by Rifampicin based assays like Xpert MTB/RIF assay and resulting in inappropriate therapy (Fasih et al., 2012).

MDR-TB by definition should be resistant to both INH and RIF with or without resistance to other first line ATT drugs. In 2010 Nepal has recorded $12.5 \%$ (Gurung et al., 2010) MDR from EPTB cases. Reports from Delhi claim $10 \%$ to $11.6 \%$ MDR positivity (Sachdeva et al., 2002; Raveendran et al., 2015). Maurya et al., reported $13.5 \%$ and 17.5\% MDR-TB positivity in 2012 and 2013 respectively from Lucknow, Uttar Pradesh (Maurya et al., 2012, 2013). NIRT (National Institute of Research in Tuberculosis), Chennai, Tamil Nadu has reported $19 \%$ of MDR among 1223 EPTB patients (Dusthackeer et al., 2005). Contrast to this, no MDR was detected in our study. As mentioned earlier, the referred patients to NIRT were mostly chronic cases/nonresponders to first line ATT.

Today over-reporting of multidrug-resistance may be due to widespread use of Xpert MTB/RIF (Denkinger et al., 2014) which considers rifampicin resistance as a surrogate marker for INH resistance. However, this assay cannot detect Isoniazid resistance and hence presence of rifampicin resistance could be either MDR or rifampicin monoresistance. Hence this surrogacy is applicable only for areas with high MDR prevalence and low prevalence of Rifampicin Mono Resistance (RMR) using Xpert MTB/RIF, but from areas or countries with increasing riampicin monoresistance it is doubtful. As per CDC recommendation, (CDC) when rifampicin resistance is detected / not detected / indeterminate in Xpert MTB/RIF assay, uniformly all EPTB/PTB specimens are to be subjected to culture and drug susceptibility testing using first line anti-TB drugs.
Rifampicin monoresistance with INH susceptibility in two strains has been observed in the present study, which would have been erroneously labeled as MDR if INH susceptibility was not performed. Automated antimycobacterial susceptibility testing of Mycobacterium tuberculosis using MGIT960 might be useful in determining drug susceptibility testing within 2 weeks' time.

To conclude it might be said that at present drug resistance is moderate and MDR absent among EPTB patients of Puducherry and neighbouring Tamil Nadu state.

\section{Acknowledgement}

We express our sincere gratitude to the Chairman, Vice-Chancellor, Dean-Research \& Allied Health Sciences, and Dean, Mahatma Gandhi Medical College \& Research Institute, SBV University, Pondicherry. We also thank Dr. Vanaja Selvakumar, Scientist F \& Deputy Director (Retired), National Institute for Research in Tuberculosis, (NIRT) Chennai, for very kindly providing us H37Rv.

\section{References}

CDC. A New Tool to Diagnose Tuberculosis: The Xpert MTB/RIF Assay. Available at:http://www.cdc.gov/tb/publications/facts heets/PDF/XpertMTB-RIFAssayFactSheet _Final.pdf.

Denkinger, C.M., Schumacher S.G., Boehme C.C., Dendukuri N., Pai M., and Steingart K.R. 2014. Xpert MTB/RIF assay for the diagnosis of extrapulmonary tuberculosis: a systematic review and meta-analysis. Eur. Respir. J. 44:435-446

Dusthackeer, A., Sekar G., Chidambaram S., Kumar V., Mehta P., and Swaminathan S. 2015. Drug resistance among extrapulmonary TB patients: Six years' experience from a supranational reference laboratory. Indian. J. Med. Res. 142:568574. 
Fasih, N., Rafiq Y., Jabeen K., and Hasan R. 2012. High Isoniazid Resistance Rates in Rifampicin Susceptible Mycobacterium tuberculosis Pulmonary Isolates from Pakistan. PLoS ONE. 7:e50551.

Gupta, K., Nair D., Sharma P., Gupta A., and Sen M.K. 2016. Changing Trends in the Susceptibility Pattern of Mycobacterium tuberculosis Over a Decade from a Tertiary Care DOTS Centre Delhi. Mycobact. Dis. 6:211

Gurung, R., Bhattacharya S.K., Pradhan B., Gurung S., and Singh Y. 2010. Phenotypic characterisation and drug sensitivity testing of mycobacteria isolated from extrapulmonary tuberculosis. Kathmandu Univ. Med. J. 8:57-61

Kandhakumari, G., and Stephen S. 2015. Extra pulmonary tuberculosis: Rapid identification of Mycobacterium tuberculosis grown in Mycobacterium growth indicator tube 960 and LowensteinJensen media, employing Standard diagnostics Bioline Mycobacterium tuberculosis protein 64 antigen detection kit. Indian. J. Med. Microbiol. 33:S122125.

Med. Res., 120:316-353.

World Health Organization (WHO). Global tuberculosis Report 2015. WHO/HTM/TB/2015.22 Geneva, Switzerland. Retrieved from:

Lee, H.Y., Lee J., Lee Y.S., Kim M.Y., Lee H.K., Lee Y.M., Shin J.H., and Ko Y. 2015. Drug-resistance pattern of Mycobacterium tuberculosis strains from patients with pulmonary and extrapulmonary tuberculosis during 2006 to 2013 in a Korean tertiary medical center. Korean. J. Intern. Med. 30:325-334.

Maurya, A. K., Singh A. K., Kumar M., Umrao J., Kant S., Nag V.L., Kushwaha R. A., and Dhole T.N. 2013. Changing patterns and trends of multidrug-resistant tuberculosis at referral centre in Northern India: a 4-year experience. Indian. J. Med. Microbiol. 31:40-46.

Maurya, A.K., Kant S., Nag V.L., Kushwaha R.A., and Dhole T.N. 2012. Trends of antituberculosis drug resistance pattern in new cases and previously treated cases of extrapulmonary tuberculosis cases in referral hospitals in northern India. $J$. Postgrad. Med. 58:185-189.

Mittal, N., and Bansal P. 2014. Multidrug resistant extrapulmonary tuberculosis - three case reports and review of literature. Intern. Med. Inside. 2:2. http://dx.doi.org/10.7243/2052-6954-2-2

Raveendran, R., Oberoi J. K., and Wattal C. 2015. Multidrug-resistant pulmonary and extrapulmonary tuberculosis: a 13 years retrospective hospital-based analysis. Indian. J. Med. Res. 142:575-582.

Sachdeva, R., Gardre D.V., and Talwar V. 2002. Characterization and drug susceptibility patterns of extra-pulmonary mycobacterial isolates. Indian. J. Med. Res. 115:102-105.

Sharma, S. K., and Mohan, A. 2004. Extrapulmonary tuberculosis. Indian. $J$. http://apps.who.int/iris/bitstream/10665/191 102/1/9789241565059_eng.pdf

Yadav, S., and Rawal G. 2016. Primary Extrapulmonary Multidrug-Resistant Tuberculosis of the Sternum without HIV Infection. J. Clin. Diagn. Res. 10:RD01RD03.

\section{How to cite this article:}

Gandhi Kandhakumari and Selvaraj Stephen. 2017. 'Drug Susceptibility Pattern of Mycobacterium tuberculosis Isolates from Extrapulmonary Tuberculosis Cases in Puducherry and Surrounding Tamil Nadu, South India'. Int.J.Curr.Microbiol.App.Sci. 6(10): 3557-3561. doi: https://doi.org/10.20546/ijcmas.2017.610.419 